\title{
Patterns of Long COVID Symptoms: A Multi-Center Cross Sectional Study
}

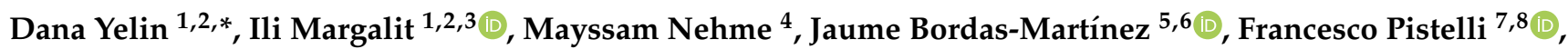 \\ Dafna Yahav 2,3 , Idris Guessous ${ }^{4,9}$, Xavier Durà-Miralles ${ }^{9,10}$, Laura Carrozzi ${ }^{6}$, Irit Shapira-Lichter ${ }^{2,11}$ (1), \\ Pauline Vetter ${ }^{12}$, Dolores Peleato-Catalan ${ }^{13}$, Giusy Tiseo ${ }^{14}$, Eytan Wirtheim ${ }^{15}$, Laurent Kaiser ${ }^{12}$, \\ Carlota Gudiol ${ }^{6,16,17}$ (1) , Marco Falcone ${ }^{14}$, Leonard Leibovici ${ }^{18}$ and on behalf of the LongCOV Research Group ${ }^{\dagger}$
}

\section{check for}

updates

Citation: Yelin, D.; Margalit, I.;

Nehme, M.; Bordas-Martínez, J.;

Pistelli, F.; Yahav, D.; Guessous, I.;

Durà-Miralles, X.; Carrozzi, L.;

Shapira-Lichter, I.; et al. Patterns of

Long COVID Symptoms: A

Multi-Center Cross Sectional Study. J.

Clin. Med. 2022, 11, 898.

https://doi.org/10.3390/

jcm11040898

Academic Editor: Danilo Buonsenso

Received: 12 January 2022

Accepted: 4 February 2022

Published: 9 February 2022

Publisher's Note: MDPI stays neutral with regard to jurisdictional claims in published maps and institutional affiliations.

Copyright: (C) 2022 by the authors. Licensee MDPI, Basel, Switzerland. This article is an open access article distributed under the terms and conditions of the Creative Commons Attribution (CC BY) license (https:// creativecommons.org/licenses/by/ $4.0 /)$.
1 COVID Recovery Clinic, Rabin Medical Center, Beilinson Hospital, Petah-Tikva 4941492, Israel; ilimargalit@gmail.com

2 Faculty of Medicine, Tel Aviv University, Ramat Aviv, Tel Aviv 6910203, Israel; dafna.yahav@gmail.com (D.Y.); iritlichter@yahoo.com (I.S.-L.)

3 Infectious Diseases Unit, Rabin Medical Center, Beilinson Hospital, Petah-Tikva 4941492, Israel

4 Division and Department of Primary Care Medicine, Geneva University Hospitals, 1211 Geneva, Switzerland; Mayssam.Nehme@hcuge.ch (M.N.); Idris.Guessous@hcuge.ch (I.G.)

5 Pulmonology Department, Bellvitge University Hospital, 08907 Barcelona, Spain; jbordas@bellvitgehospital.cat

6 Infectious Diseases Department, Bellvitge University Hospital, Institute for Biomedical Research (IDIBELL), University of Barcelona, Hospitalet de Llobregat, 08907 Barcelona, Spain; laura.carrozzi@unipi.it (L.C.); carlotagudiol@gmail.com (C.G.)

7 Department of Surgical, Medical, and Molecular Pathology and Critical Care Medicine, University of Pisa, 56126 Pisa, Italy; f.pistelli@ao-pisa.toscana.it

8 Pulmonary Unit, Cardiothoracic and Vascular Department, University Hospital, 56124 Pisa, Italy

9 Faculty of Medicine, University of Geneva, 1205 Geneva, Switzerland; xavierduramiralles@gmail.com

10 Spanish Network for Research in Infectious Diseases (REIPI), Instituto de Salud Carlos III, 08003 Madrid, Spain

11 Functional MRI Center, Rabin Medical Center, Beilinson Hospital, Petah-Tikva 4941492, Israel

12 Division of Infectious Diseases, Geneva University Hospitals, 1211 Geneva, Switzerland; Pauline.Vetter@hcuge.ch (P.V.); Laurent.Kaiser@hcuge.ch (L.K.)

13 Ramona via Primary Care Center, El Prat de Llobregat, 08820 Barcelona, Spain; mpeleato@ambitcp.catsalut.net

14 Infectious Diseases Unit, Department of Clinical and Experimental Medicine, University of Pisa, 56126 Pisa, Italy; tiseogiusy@gmail.com (G.T.); marco.falcone@unipi.it (M.F.)

15 Management, Rabin Medical Center, Petah-Tikva 4941492, Israel; eytanwir@clalit.org.il

16 Infectious Diseases Unit, Hospital Duran y Reynals, Institut Català d'Oncologia (ICO), IDIBELL, 08908 Barcelona, Spain

17 Centro de Investigación Biomédica en Red de Enfermedades Infecciosas (CIBERINFEC), Instituto de Salud Carlos III, 08003 Madrid, Spain

18 Research Authority, Rabin Medical Center, Beilinson Hospital, Petah-Tikva 4941492, Israel; leibovic@tauex.tau.ac.il

* Correspondence: dana.yelin@gmail.com; Tel.: +972-3-9377728; Fax: +972-3-9376512

+ Membership of the LongCOV Research Group is provided in Acknowledgments part.

Abstract: Background: Long COVID has become a burden on healthcare systems worldwide. Research into the etiology and risk factors has been impeded by observing all diverse manifestations as part of a single entity. We aimed to determine patterns of symptoms in convalescing COVID-19 patients. Methods: Symptomatic patients were recruited from four countries. Data were collected regarding demographics, comorbidities, acute disease and persistent symptoms. Factor analysis was performed to elucidate symptom patterns. Associations of the patterns with patients' characteristics, features of acute disease and effect on daily life were sought. Results: We included 1027 symptomatic post-COVID individuals in the analysis. The majority of participants were graded as having a non-severe acute COVID-19 ( $\mathrm{N}=763,74.3 \%)$. We identified six patterns of symptoms: cognitive, pain-syndrome, pulmonary, cardiac, anosmia-dysgeusia and headache. The cognitive pattern was the major symptoms pattern, explaining $26.2 \%$ of the variance; the other patterns each explained $6.5-9.5 \%$ of the variance. The cognitive pattern was higher in patients who were outpatients during the acute disease. The pain-syndrome pattern was associated with acute disease severity, higher 
in women and increased with age. The pulmonary pattern was associated with prior lung disease and severe acute disease. Only two of the patterns (cognitive and cardiac) were associated with failure to return to pre-COVID occupational and physical activity status. Conclusion: Long COVID diverse symptoms can be grouped into six unique patterns. Using these patterns in future research may improve our understanding of pathophysiology and risk factors of persistent COVID, provide homogenous terminology for clinical research, and direct therapeutic interventions.

Keywords: COVID-19; post-COVID; long lasting symptoms; SARS-CoV-2

\section{Background}

Persistent symptoms following coronavirus disease 2019 (COVID-19) have been described in a significant percentage of post-COVID individuals. The only established correlates identified thus far are the severity of the acute disease and a higher preponderance in women [1-4]. Other potential risk factors such as age have been described in some studies, but not in others $[1,3,5]$.

Similar to the acute disease, which manifests in multiple ways, post-COVID individuals report a multitude of symptoms, involving different organ systems [6]. Whereas, in the acute disease, several pathophysiological mechanisms have already been identified (i.e., direct viral induced damage, microthrombi and immune mediated damage), the underlying mechanisms behind long COVID are still unknown and are likely to be multifactorial. Aggregating all individuals with long COVID under a single entity might be misleading for our understanding of the pathophysiology, risk factors and potential interventions for these individuals. Assigning these symptoms to unique patterns might advance future research and a personalized treatment approach [7].

In this study, we aimed to determine patterns of symptoms among convalescing COVID-19 individuals.

\section{Methods}

\subsection{Study Design, Participants and Data Collection}

The study was a multi-center cohort study, conducted in the multi-disciplinary longCOVID clinics in four countries (Israel, Switzerland, Spain and Italy). Data were collected either at the first clinic visit (Israel, Italy, Spain) or by telephone interview (Switzerland, Spain). Adult (age $\geq 18$ years) individuals visiting the clinics between May 2020 and March 2021 reporting on at least one persisting symptom, at least 30 days after a PCR-proven diagnosis of COVID-19 were consecutively included (for additional information regarding the clinics and respective structured follow-up programs-see Supplementary Material). Each patient was interviewed, using a structured questionnaire (grading 14 symptoms subjectively according to severity on a $0-3$ scale, representing no, mild, moderate and severe impact on daily life respectively). Graded symptoms were: fatigue, dyspnea, cough, chest pain, palpitations, insomnia, memory impairment, concentration difficulties, myalgia, arthralgia, paresthesia (numbness or tingling), headache, anosmia/dysgeusia, and emotional distress. Data regarding part of the patients from the Swiss cohort were previously reported [8]. Pulmonary function testing was completed in three of the four centers (Israel, Spain, Italy).

The study was approved by the local ethics committee of each participating center (approval numbers: Israel 0458-20-RMC; Italy CEAVNO n. 1768; Spain PR374/20; Switzerland CER 2020-01273). All patients signed or gave oral informed consent prior to participation.

\subsection{Statistical Analysis}

A principal component analysis of the 14 reported symptoms was conducted in order to identify key patterns of symptoms. We used a varimax rotation. The symptoms were tested for factorability using correlates above 0.3 and Kaiser-Meyer-Olkin measure of 
sampling adequacy of over 0.6. Eigenvalues above 0.9 were used to select the number of factors. Loading of 0.4 and up was considered as significant.

We looked for associations of the patterns with the patients' age, gender, underlying comorbidities, and features of the acute disease (hospitalization, severe disease according to WHO classification [9], and acute symptoms). We also examined whether the factors were related to return to work and return to the pre-infection level of physical activity. Student's $t$-tests and analysis of variance (ANOVA) were used for comparing the values of the factors across categories; Spearman's rank correlation coefficient was implemented for testing the correlation of factors with continuous variables. We used the Bonferroni correction to adjust $p$ values for multiple hypotheses and reported the adjusted $p$ values. Data analysis was undertaken using IBM SPSS version 27 (Armonk, New York, NY, USA).

\section{Results}

From the four centers, 1737 participants were recruited. Out of these, 1033 (59.5\%) reported ongoing symptoms, however, 6 had incomplete responses regarding one or more of the 14 symptoms and were excluded. In total, 1027 persistently symptomatic adult patients were included in the study (see Supplemental Figure S1). The median interval from onset of acute COVID-19 to the interview was 123 days (interquartile range 80-204 days). Included patients had acute COVID-19 from March 2020 to March 2021. Five hundred fifty nine participants $(54.4 \%)$ were women and $337(32.8 \%)$ were hospitalized during the acute disease. Patient characteristics and the prevalence of each persistent symptom in this cohort are presented in Table 1 . Three hundred twenty six individuals $(31.7 \%)$ reported worse physical activity status compared to pre-infection state, and 131 (of the 735 for whom data were available, $17.8 \%$ ) were unable to return to their former work because of ongoing symptoms at the time of the clinic visit.

Using factor analysis, we identified 6 different patterns of the 14 symptoms (Figure 1, for exact loading data see Supplemental Table S1). The first was a cognitive-emotional pattern combining complaints of impaired concentration, memory loss, emotional distress and insomnia. The second was indicative of pain manifestations: myalgia, arthralgia and, to a lesser extent, paresthesias and fatigue. The third was a pulmonary pattern, combining dyspnea, cough and chest pain. The fourth pattern (cardiac) consisted of a combination of palpitations or tachycardia with chest pain. The fifth and sixth patterns were anosmia and/or dysgeusia and isolated headache, respectively. The cognitiveemotional pattern was the major symptoms pattern, explaining $26.2 \%$ of the variance; each of the other patterns explained 6.5-9.5\% of the variance. Cumulatively, all patterns explained $64.6 \%$ of the variance. On four sensitivity analyses, excluding one of the centers successively, similar patterns were achieved each time; the differences manifested in the order of the factors only.

Associations of the various patterns with demographics, comorbidities and manifestations of the acute disease are detailed in Table 2. The cognitive-emotional pattern was associated with features of the acute disease: outpatient treatment (mean 0.1 (standard deviation 1.03) vs. $-0.15(1.00), p<0.001)$, fatigue $(0.25(1.13)$ vs. $-0.37(0.75)$, $p<0.001)$, myalgia $(0.29(1.18)$ vs. $-0.22(0.84), p<0.001)$ and anosmia $(0.34$ (1.17 vs. $-0.18(0.90), p<0.001)$. The pain-syndrome pattern was associated with the severity of the acute disease $(0.22(0.98)$ vs. $-0.08(1.00), p<0.001)$ and was higher in women $(0.11(1.08)$ vs. $-0.13(0.88), p<0.001)$. The pulmonary pattern was associated with prior lung disease $(0.41(1.24)$ vs. $-0.03(0.97), p<0.001)$, severe acute disease $(0.20(0.98)$ vs. $-0.07(0.97), p<0.001)$, inpatient treatment $(0.17(0.98)$ vs. $-0.07(1.01), p<0.001)$, and dyspnea $(0.33(1.16$ vs. $-0.16(0.85), p<0.001)$ during the acute disease. Higher values in the pulmonary pattern were also associated with abnormal pulmonary function testing (diffusing capacity of carbon monoxide or total lung capacity below $80 \%$ of expected and FEV1 to FVC ratio below 0.7). The cardiac, anosmia and headache patterns were associated with age younger than 65 years $(0.06(1.05)$ vs. $-0.28(0.63) ; 0.03(1.04)$ vs. -0.17 (0.74); and 0.04 (1.04) vs. $-0.19(0.72)$, respectively, $p<0.001$ for all comparisons). 
The pain-syndrome, pulmonary and cardiac patterns were inversely correlated with the time elapsed between the acute disease and clinic visit (Spearman's rho $-0.149,-0.122$, and -0.225 , respectively, $p<0.001$ for all comparisons).

Table 1. Comparison of patient characteristics from the different centers.

\begin{tabular}{|c|c|c|c|c|c|}
\hline & All & Israel & Switzerland & Spain & Italy \\
\hline No. of patients, $\mathrm{N}(\%)$ & 1027 & $544(53)$ & $256(24.9)$ & $115(11.2)$ & $112(10.9)$ \\
\hline Gender-women, N (\%) & $559(54.4)$ & $307(56.4)$ & $144(56.3)$ & $60(52.2)$ & $48(42.9)$ \\
\hline Age, mean (SD) & $49.2(16.1)$ & $46.4(15.5)$ & $45.5(15.1)$ & $58.4(12.4)$ & $61.2(15.5)$ \\
\hline BMI, mean (SD) & $28(5.6)$ & $27.6(5.6)$ & $28.7(7.7)$ & $29.8(6.1)$ & $28.2(4.6)$ \\
\hline Obese, N (\%) & $223(30.2)$ & $149(29.0)$ & $3(15.7)$ & $42(42.4)$ & $29(26.1)$ \\
\hline${ }^{*}$ missing 280 & $\mathrm{~N}=738$ & $\mathrm{~N}=518$ & $\mathrm{~N}=19$ & $\mathrm{~N}=99$ & $\mathrm{~N}=111$ \\
\hline $\begin{array}{l}\text { Smoking, } N(\%) \\
\quad * \text { missing } 79\end{array}$ & $255(24.8)$ & $132(24.3)$ & $59(23.0)$ & $41(35.7)$ & $23(20.5)$ \\
\hline Diabetes, N (\%) & $92(9.0)$ & $45(8.3)$ & $10(3.9)$ & $22(19.1)$ & $15(13.4)$ \\
\hline Hypertension, N (\%) & $206(20.1)$ & $89(16.4)$ & $27(10.5)$ & $50(43.5)$ & $40(35.7)$ \\
\hline IHD, N (\%) & $52(5.1)$ & $25(4.6)$ & $17(6.6)$ & $1(0.9)$ & $9(8.0)$ \\
\hline Prior lung disease, N (\%) & $79(7.7)$ & $35(6.4)$ & $19(7.4)$ & $16(13.9)$ & $9(8.0)$ \\
\hline $\begin{array}{l}\text { Hospitalization, } \mathrm{N}(\%) \\
{ }^{*} \text { Missing } 78\end{array}$ & $337(32.8)$ & $122(22.4)$ & $26(10.2)$ & 79 (68.7) & $112(100)$ \\
\hline Severe disease ${ }^{a}, N(\%)$ & $264(25.7)$ & $86(15.8)$ & $26(10.2)$ & $72(62.6)$ & $80(71.4)$ \\
\hline $\begin{array}{l}\text { Time to clinic visit (days), median (IQR) } \\
{ }^{*} \text { Missing } 26\end{array}$ & $123(80-204)$ & $97(66-130)$ & 217 (203-233) & $115(87-224)$ & $146(127-185)$ \\
\hline Fatigue $\mathrm{b}, \mathrm{N}(\%)$ & $691(67.2)$ & $411(75.6)$ & $129(50.4)$ & $90(78.3)$ & $61(54.5)$ \\
\hline Dyspnea $^{\mathrm{b}}, \mathrm{N}(\%)$ & $480(46.7)$ & $277(50.9)$ & $62(24.2)$ & $85(73.9)$ & $56(50.0)$ \\
\hline Cough $\mathrm{b}, \mathrm{N}(\%)$ & $187(18.2)$ & $108(19.9)$ & $21(8.2)$ & $35(30.4)$ & $23(20.5)$ \\
\hline Chest pain ${ }^{b}, N(\%)$ & $204(19.9)$ & $166(30.5)$ & $14(5.5)$ & $24(20.9)$ & $0(0)$ \\
\hline Palpitations ${ }^{b}, \mathrm{~N}(\%)$ & $104(10.1)$ & $67(12.3)$ & $18(7.0)$ & $19(16.5)$ & $0(0)$ \\
\hline Myalgia b,$N(\%)$ & $332(32.3)$ & $204(37.5)$ & $37(14.5)$ & $46(40.0)$ & $45(40.2)$ \\
\hline Arthralgia ${ }^{b}, N(\%)$ & $157(15.3)$ & $51(9.4)$ & $23(9.0)$ & $33(28.7)$ & $50(44.6)$ \\
\hline Parasthesias ${ }^{b}, \mathrm{~N}(\%)$ & $193(18.8)$ & $109(20.0)$ & $13(5.1)$ & $32(27.8)$ & $39(34.8)$ \\
\hline Insomnia ${ }^{b}, \mathrm{~N}(\%)$ & $259(25.2)$ & $204(37.5)$ & $27(10.5)$ & $28(24.3)$ & $0(0)$ \\
\hline Headache $^{\mathrm{b}}, \mathrm{N}(\%)$ & $153(14.9)$ & $69(12.7)$ & $44(17.2)$ & $24(20.9)$ & $16(14.3)$ \\
\hline Memory loss ${ }^{b}, N(\%)$ & $315(30.7)$ & $201(36.9)$ & $35(13.7)$ & $33(28.7)$ & $46(41.1)$ \\
\hline Concentration impairment ${ }^{b}, N(\%)$ & $312(30.4)$ & $212(39.0)$ & $31(12.1)$ & $26(22.6)$ & $43(38.4)$ \\
\hline Anosmia/dysguesia ${ }^{b}, \mathrm{~N}(\%)$ & $289(28.2)$ & $160(29.4)$ & $70(27.3)$ & $24(20.9)$ & $30(26.8)$ \\
\hline $\begin{array}{l}\text { Worse physical activity status }{ }^{\mathrm{c}}, \mathrm{N}(\%) \\
{ }^{*} \text { missing } 42\end{array}$ & $326(31.7)$ & $142(26.1)$ & $134(52.3)$ & $43(37.4)$ & $7(6.3)$ \\
\hline $\begin{array}{l}\text { Worse employment status }{ }^{\mathrm{c}}, \mathrm{N}(\%) \\
{ }^{*} \text { missing } 292\end{array}$ & $131(12.8)$ & $102(18.8)$ & 一 & $22(19.1)$ & $7(6.3)$ \\
\hline Pulmonary function testing performed, N (\%) & $665(64.8)$ & $517(95.0)$ & 一 & $51(44.3)$ & $97(86.6)$ \\
\hline
\end{tabular}

Abbreviations: BMI—body mass index; IHD—ischemic heart disease, IQR —interquartile range, SD—standard deviation. ${ }^{\mathrm{a}}$ : According to WHO severity grading—classified as severe and critical [8]. ${ }^{\mathrm{b}}$ : Prevalence of persistent symptom at clinic visit, regardless of severity. ${ }^{c}$ : Compared to the pre-acute disease status. ${ }^{*}$ Missing data on baseline characteristic.

High values on the cognitive-emotional and cardiac patterns were associated with failure to return to baseline occupational status (mean 0.49 (SD 1.26) vs. -0.04 (0.99) $p<0.001 ; 0.44$ (1.29) vs. $0.02(0.99), p=<0.001$ respectively). These two patterns were also associated with worsened physical activity status compared to pre-infection state (0.18 (1.10) vs. -0.09 (0.94), $p<0.001 ; 0.18$ (1.18) vs. -0.08 (0.91), $p=<0.001$, respectively), while the anosmia pattern showed an inverse association with physical activity status compared to pre-infection state, implying improved physical status (-0.12 (1.02) vs. 0.06 (0.99), $p=0.007)$. These associations are presented in Table 3. 


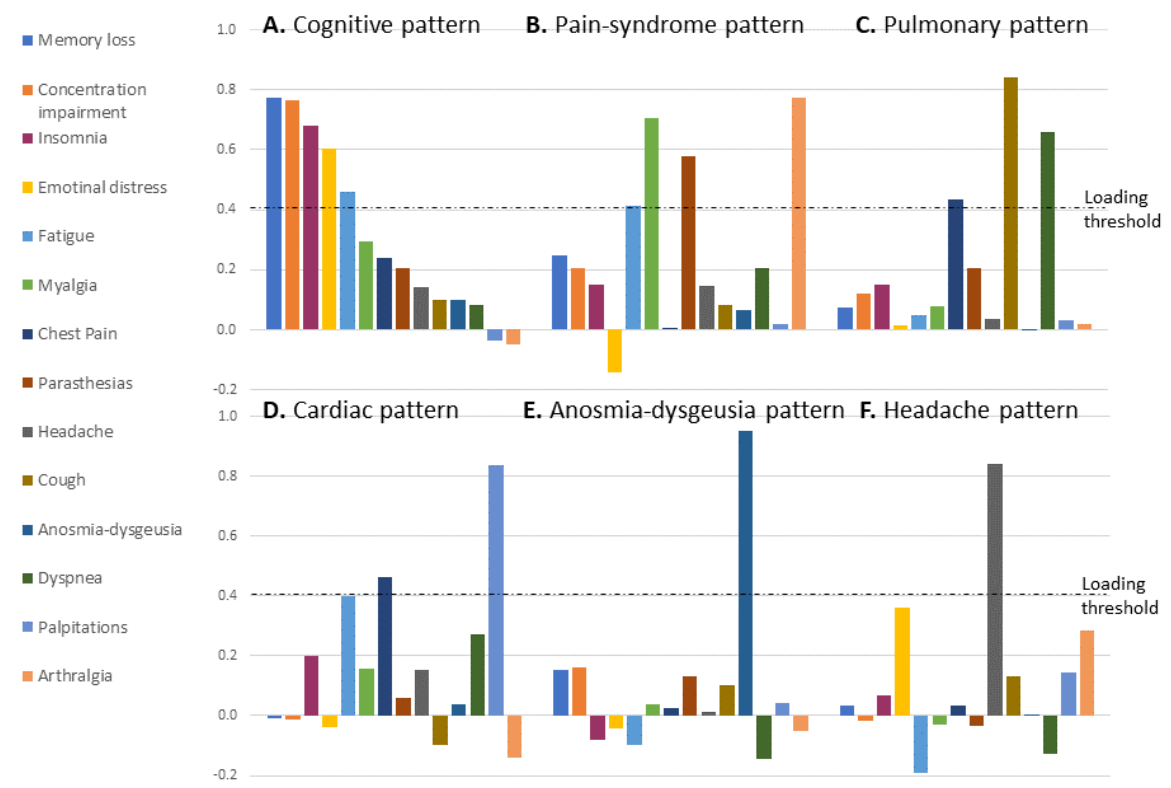

Figure 1. Loading of the different symptoms in each symptom pattern. (A) Cognitive-emotional pattern combining impaired cognitive functions, emotional distress, insomnia and fatigue (explaining $26.2 \%$ of variance); (B) Pain syndrome pattern combining myalgia, arthralgia, paresthesias and fatigue (9.5\% of variance) (C) Pulmonary pattern combining dyspnea, cough and chest pain ( $8.5 \%$ of variance); (D) Cardiac — combining palpitations or tachycardia with chest pain (7.1\% of variance), fatigue with a loading of 0.39 is under the threshold; (E) Anosmia-dysgeusia pattern-isolated anosmia and dysgeusia (6.8\% of variance); (F) Headache pattern-isolated headache (6.5\% of variance); The 6 factors in total explain $64.6 \%$ of variance between the recoverees; KMO: 0.811, Bartlett's test of sphericity chi-square: $2682.51, p<0.001$.

Table 2. Patterns associations with selected demographics, comorbidities and features of the acute disease. Means for each of the patterns with standard deviation in parenthesis are shown. Significant associations ${ }^{a}$ marked in bold. Analysis by $t$-test (Spearman where indicated).

\begin{tabular}{|c|c|c|c|c|c|c|c|}
\hline & $\begin{array}{l}\text { Feature } \\
\text { N (\%) }\end{array}$ & $\begin{array}{c}\text { Cognitive-Emotional } \\
\text { Pattern }\end{array}$ & $\begin{array}{l}\text { Pain Syndrome } \\
\text { Pattern }\end{array}$ & $\begin{array}{l}\text { Pulmonary } \\
\text { Pattern }\end{array}$ & $\begin{array}{l}\text { Palpitations-Chest } \\
\text { Pain Pattern }\end{array}$ & $\begin{array}{c}\text { Anosmia-Dysgeusia } \\
\text { Pattern }\end{array}$ & $\begin{array}{l}\text { Headache } \\
\text { Pattern }\end{array}$ \\
\hline \multirow[t]{2}{*}{ Gender } & $\begin{array}{l}\text { Women } \\
559(54.5)\end{array}$ & $0.08(1.05)$ & $0.11(1.08)$ & $0.03(1.06)$ & $0.04(1.03)$ & $0.05(1.04)$ & $0.13(1.13)$ \\
\hline & $\begin{array}{c}\text { Men } \\
466(45.5)\end{array}$ & $-0.10(0.93)$ & $-0.13(0.88)$ & $-0.04(0.93)$ & $-0.05(0.96)$ & $-0.06(0.94)$ & $-0.15(0.79)$ \\
\hline \multirow{3}{*}{ Age } & $\begin{array}{l}\geq 65 \text { years } \\
168(16.4)\end{array}$ & $-0.17(0.95)$ & $0.09(0.86)$ & $0.06(1.01)$ & $-0.28(0.63)$ & $-0.17(0.74)$ & $-0.19(0.72)$ \\
\hline & $\begin{array}{l}<65 \text { years } \\
858(83.6)\end{array}$ & $0.03(1.01)$ & $-0.02(1.02)$ & $-0.01(1.00)$ & $0.06(1.05)$ & $0.03(1.04)$ & $0.04(1.04)$ \\
\hline & Rho $(p)^{\mathrm{b}}$ & $-0.05(0.12)$ & $0.15(<0.001)$ & $0.03(0.34)$ & $-0.12(<0.001)$ & $-0.06(0.06)$ & $\begin{array}{r}-0.09 \\
(0.006)\end{array}$ \\
\hline \multirow{2}{*}{ BMI } & $\begin{array}{c}\geq 30 \\
226(30.2)\end{array}$ & $0.07(1.09)$ & $0.13(0.95)$ & $0.11(1.09)$ & 0.09 (1.07) & $-0.06(0.97)$ & $-0.17(0.98)$ \\
\hline & $\begin{array}{c}<30 \\
521(69.8)\end{array}$ & $0.02(1.02)$ & $0.10(0.99)$ & $0.06(0.99)$ & $0.04(1.02)$ & $-0.02(0.91)$ & $-0.15(0.86)$ \\
\hline \multirow{3}{*}{$\begin{array}{l}\text { Smoker } \\
\text { (ever) }\end{array}$} & Rho $(p)^{\mathrm{b}}$ & $-0.02(0.61)$ & $0.04(0.28)$ & $0.04(0.29)$ & $0.01(0.97)$ & $-0.08(0.03)$ & $-0.05(0.16)$ \\
\hline & $\begin{array}{c}\text { Yes } \\
255(26.9)\end{array}$ & $0.01(1.04)$ & $0.08(1.04)$ & $-0.02(0.99)$ & $0.04(1.00)$ & $0.00(0.98)$ & $-0.03(0.98)$ \\
\hline & $\begin{array}{c}\text { No } \\
693(73.1)\end{array}$ & $0.02(0.99)$ & $-0.10(0.93)$ & $0.13(1.05)$ & $-0.05(1.05)$ & $0.00(1.00)$ & $-0.04(1.04)$ \\
\hline \multicolumn{8}{|c|}{ Comorbidities } \\
\hline \multirow{2}{*}{$\begin{array}{l}\text { Prior lung } \\
\text { disease }\end{array}$} & $\begin{array}{c}\text { Yes } \\
79(7.7)\end{array}$ & $-0.01(1.11)$ & $-0.04(0.94)$ & $0.41(1.24)$ & $-0.08(0.82)$ & $-0.10(1.02)$ & $-0.08(1.00)$ \\
\hline & $\begin{array}{c}\text { No } \\
948(92.3)\end{array}$ & $0.00(0.99)$ & $0.00(1.01)$ & $-0.03(0.97)$ & $0.01(1.01)$ & $0.01(1.00)$ & $0.01(1.00)$ \\
\hline \multirow[t]{2}{*}{ Diabetes } & $\begin{array}{c}\text { Yes } \\
92(9.0)\end{array}$ & $-0.03(1.03)$ & $0.23(0.97)$ & $0.20(1.14)$ & $-0.01(0.96)$ & $-0.17(0.80)$ & $-0.27(0.82)$ \\
\hline & $\begin{array}{c}\text { No } \\
926(91.0)\end{array}$ & $0.01(1.00)$ & $-0.02(1.00)$ & $-0.02(0.99)$ & $0.00(1.01)$ & $0.02(1.02)$ & $0.03(1.02)$ \\
\hline
\end{tabular}


Table 2. Cont.

\begin{tabular}{|c|c|c|c|c|c|c|c|}
\hline & $\begin{array}{l}\text { Feature } \\
\text { N (\%) }\end{array}$ & $\begin{array}{c}\text { Cognitive-Emotional } \\
\text { Pattern }\end{array}$ & $\begin{array}{c}\text { Pain Syndrome } \\
\text { Pattern }\end{array}$ & $\begin{array}{l}\text { Pulmonary } \\
\text { Pattern }\end{array}$ & $\begin{array}{l}\text { Palpitations-Chest } \\
\text { Pain Pattern }\end{array}$ & $\begin{array}{c}\text { Anosmia-Dysgeusia } \\
\text { Pattern }\end{array}$ & $\begin{array}{l}\text { Headache } \\
\text { Pattern }\end{array}$ \\
\hline \multirow[t]{2}{*}{ HTN } & $\begin{array}{c}\text { Yes } \\
206(20.2)\end{array}$ & $-0.07(0.98)$ & $0.10(0.94)$ & $0.12(1.02)$ & $-0.12(0.82)$ & $-0.10(0.93)$ & $-0.21(0.78)$ \\
\hline & $\begin{array}{c}\text { No } \\
812(79.8)\end{array}$ & $0.02(1.01)$ & $-0.02(1.02)$ & $-0.03(1.00)$ & $0.04(1.04)$ & $0.03(1.02)$ & $0.05(1.05)$ \\
\hline \multirow{2}{*}{ IHD } & $\begin{array}{c}\text { Yes } \\
52(5.1)\end{array}$ & $0.14(1.27)$ & $-0.13(0.77)$ & $0.11(1.05)$ & $-0.22(0.66)$ & $-0.14(1.02)$ & $0.02(1.25)$ \\
\hline & $\begin{array}{c}\text { No } \\
966(94.9)\end{array}$ & $-0.01(0.99)$ & $0.01(1.01)$ & $0.00(1.00)$ & $0.01(1.02)$ & $0.01(1.00)$ & $0.00(0.99)$ \\
\hline \multirow{2}{*}{$\begin{array}{c}\text { Atrial } \\
\text { fibrillation }\end{array}$} & $\begin{array}{c}\text { Yes } \\
27(2.6)\end{array}$ & $-0.33(0.99)$ & $0.12(0.68)$ & $0.00(0.67)$ & $-0.33(0.44)$ & $-0.33(0.54)$ & $-0.20(0.72)$ \\
\hline & $\begin{array}{c}\text { No } \\
1000(97.4)\end{array}$ & $0.01(1.00)$ & $0.00(1.01)$ & $0.00(1.01)$ & $0.01(1.01)$ & $0.01(1.01)$ & $0.01(1.01)$ \\
\hline \multicolumn{8}{|c|}{ Acute COVID-19 features } \\
\hline \multirow[t]{2}{*}{ Hospitalization } & $\begin{array}{c}\text { Yes } \\
337(35.5)\end{array}$ & $-0.15(1.00)$ & $0.15(0.95)$ & $0.17(0.98)$ & $-0.11(0.95)$ & $-0.16(0.79)$ & $-0.17(0.82)$ \\
\hline & $\begin{array}{c}\text { No } \\
612(64.5)\end{array}$ & $0.10(1.03)$ & $-0.02(1.04)$ & $-0.07(1.01)$ & 0.09 (1.08) & $0.07(1.07)$ & $0.04(1.07)$ \\
\hline \multirow{2}{*}{$\begin{array}{l}\text { Abnormal } \\
\text { CXR }\end{array}$} & $\begin{array}{c}\text { Yes } \\
140(39.5)\end{array}$ & $-0.14(1.05)$ & $0.17(0.96)$ & $0.31(1.06)$ & $-0.04(0.94)$ & $-0.25(0.72)$ & $-0.10(0.95)$ \\
\hline & $\begin{array}{c}\text { No } \\
214(60.5)\end{array}$ & $-0.07(1.05)$ & $0.40(1.12)$ & $0.12(1.00)$ & $0.04(1.12)$ & $0.04(0.99)$ & $-0.07(0.92)$ \\
\hline \multirow{2}{*}{$\begin{array}{l}\text { WHO } \\
\text { severity }\end{array}$} & $\begin{array}{c}\text { Severe } \\
264(25.6)\end{array}$ & $-0.16(0.99)$ & $0.22(0.98)$ & $0.20(1.07)$ & $-0.08(0.94)$ & $-0.19(0.78)$ & $-0.13(0.86)$ \\
\hline & $\begin{array}{l}\text { Non-severe } \\
763(74.4)\end{array}$ & $0.05(1.00)$ & $-0.08(1.00)$ & $-0.07(0.97)$ & $0.03(1.02)$ & $0.07(1.06)$ & $0.05(1.04)$ \\
\hline \multicolumn{8}{|c|}{ Symptoms of the acute disease } \\
\hline \multirow[t]{2}{*}{ Fatigue $^{c}$} & $\begin{array}{c}\text { Yes } \\
529(69.0)\end{array}$ & $0.25(1.13)$ & $0.19(1.08)$ & $0.12(1.09)$ & $0.22(1.12)$ & $0.00(1.02)$ & $-0.17(0.97)$ \\
\hline & $\begin{array}{c}\text { No } \\
209(31.0)\end{array}$ & $-0.37(0.75)$ & $0.03(0.88)$ & $0.04(0.98)$ & $-0.19(0.87)$ & $-0.03(0.80)$ & $-0.11(0.78)$ \\
\hline \multirow[t]{2}{*}{ Dyspnea $^{c}$} & $\begin{array}{c}\text { Yes } \\
405(54.5)\end{array}$ & $0.12(1.13)$ & $0.24(1.09)$ & $0.33(1.16)$ & $0.19(1.15)$ & $-0.03(0.98)$ & $-0.14(0.97)$ \\
\hline & $\begin{array}{c}\text { No } \\
338(45.5)\end{array}$ & $-0.02(0.99)$ & $0.04(0.94)$ & $-0.16(0.85)$ & $-0.01(0.96)$ & $0.01(0.94)$ & $-0.15(0.86)$ \\
\hline \multirow[t]{2}{*}{ Anosmia $^{c}$} & $\begin{array}{c}\text { Yes } \\
360(49.2)\end{array}$ & $0.34(1.17)$ & $0.13(1.05)$ & $0.12(1.09)$ & $0.21(1.04)$ & $0.36(1.16)$ & $-0.23(0.92)$ \\
\hline & $\begin{array}{c}\text { No } \\
371(50.8)\end{array}$ & $-0.18(0.90)$ & $0.15(1.00)$ & $0.09(1.04)$ & $0.00(1.10)$ & $-0.34(0.54)$ & $-0.05(0.93)$ \\
\hline \multirow[t]{2}{*}{ Myalgia $^{c}$} & $\begin{array}{c}\text { Yes } \\
418(56.6)\end{array}$ & $0.29(1.18)$ & $0.37(1.15)$ & $0.16(1.16)$ & 0.19 (1.03) & $0.02(1.01)$ & $-0.14(1.06)$ \\
\hline & $\begin{array}{c}\text { No } \\
321(43.4)\end{array}$ & $-0.22(0.84)$ & $-0.14(0.75)$ & $0.03(0.92)$ & $-0.02(1.10)$ & $-0.05(0.89)$ & $-0.15(0.71)$ \\
\hline $\begin{array}{l}\text { Time to } \\
\text { clinic visit } \\
\text { rho }(p)\end{array}$ & $\mathrm{N}=1001$ & $-0.04(0.204)$ & $-0.149(<0.001)$ & $\begin{array}{r}-0.122 \\
(<0.001)\end{array}$ & $\begin{array}{r}-0.255 \\
(<0.001)\end{array}$ & $\begin{array}{c}0.032 \\
(0.316)\end{array}$ & $\begin{array}{c}0.163 \\
(<0.001)\end{array}$ \\
\hline
\end{tabular}

Abbreviations: BMI—body mass index; CXR—chest radiogram; HTN—hypertension; IHD—ischemic heart disease; WHO-World Health Organization; ${ }^{\text {a }}: p$-value $<0.001{ }^{\text {b }}$ : Spearman's correlation with the continuous variable; ${ }^{c}$ : Symptoms of acute COVID-19 per description at clinic visit or telephone interview, regardless of severity.

Table 3. Patterns associations with patient outcomes at clinic visit. Means for each of the patterns with standard deviation in parenthesis are shown. Significant associations $(p<0.05)$ marked in bold. Analysis by $t$-test (Spearman where indicated).

\begin{tabular}{|c|c|c|c|c|c|c|c|}
\hline & $\begin{array}{l}\text { Feature } \\
\text { N (\%) }\end{array}$ & $\begin{array}{c}\text { Cognitive-Emotional } \\
\text { Pattern }\end{array}$ & $\begin{array}{l}\text { Pain Syndrome } \\
\text { Pattern }\end{array}$ & $\begin{array}{l}\text { Pulmonary } \\
\text { Pattern }\end{array}$ & $\begin{array}{l}\text { Palpitations-Chest } \\
\text { Pain Pattern }\end{array}$ & $\begin{array}{c}\text { Anosmia-Dysgeusia } \\
\text { Pattern }\end{array}$ & $\begin{array}{l}\text { Headache } \\
\text { Pattern }\end{array}$ \\
\hline \multirow{2}{*}{$\begin{array}{l}\text { Failure to } \\
\text { return to } \\
\text { physical } \\
\text { activity }\end{array}$} & $\begin{array}{c}\text { Yes } \\
326(33.1)\end{array}$ & $0.18(1.10)$ & $0.07(1.14)$ & $0.08(1.18)$ & $0.18(1.18)$ & $-0.12(1.02)$ & 0.09 (1.18) \\
\hline & $\begin{array}{c}\text { No } \\
659(66.9)\end{array}$ & $-0.09(0.94)$ & $-0.02(0.94)$ & $-0.02(0.97)$ & $-0.08(0.91)$ & $0.06(0.99)$ & $-0.05(0.90)$ \\
\hline \multirow{2}{*}{$\begin{array}{l}\text { Failure to } \\
\text { return to full } \\
\text { employment }\end{array}$} & $\begin{array}{c}\text { Yes } \\
131(17.8)\end{array}$ & $0.49(1.26)$ & $0.14(1.12)$ & $0.26(1.27)$ & $0.44(1.29)$ & $-0.02(1.01)$ & $-0.02(1.13)$ \\
\hline & $\begin{array}{c}\text { No } \\
604(82.2)\end{array}$ & $-0.04(1.00)$ & $0.15(1.00)$ & $0.06(1.00)$ & $0.02(1.00)$ & $-0.02(0.93)$ & $-0.16(0.89)$ \\
\hline \multirow{3}{*}{ DLCO } & $\begin{array}{c}\text { Abnormal }^{\mathrm{a}} \\
178(26.7)\end{array}$ & $0.13(1.15)$ & $0.14(0.90)$ & $0.38(1.18)$ & $0.16(1.21)$ & $-0.09(0.93)$ & $-0.05(1.01)$ \\
\hline & $\begin{array}{c}\text { Normal } \\
487(73.3)\end{array}$ & $0.03(1.01)$ & $0.07(0.95)$ & $-0.04(0.94)$ & $0.08(1.00)$ & $-0.02(0.92)$ & $-0.24(0.82)$ \\
\hline & Rho, $(p)^{\mathrm{b}}$ & $-0.03(0.43)$ & $-0.09(0.02)$ & $\begin{array}{c}-0.18 \\
(<0.001)\end{array}$ & $-0.05(0.23)$ & $0.13(<0.001)$ & $0.01(0.89)$ \\
\hline
\end{tabular}


Table 3. Cont.

\begin{tabular}{|c|c|c|c|c|c|c|c|}
\hline & $\begin{array}{l}\text { Feature } \\
\text { N }(\%)\end{array}$ & $\begin{array}{c}\text { Cognitive-Emotional } \\
\text { Pattern }\end{array}$ & $\begin{array}{l}\text { Pain Syndrome } \\
\text { Pattern }\end{array}$ & $\begin{array}{l}\text { Pulmonary } \\
\text { Pattern }\end{array}$ & $\begin{array}{l}\text { Palpitations-Chest } \\
\text { Pain Pattern }\end{array}$ & $\begin{array}{c}\text { Anosmia-Dysgeusia } \\
\text { Pattern }\end{array}$ & $\begin{array}{l}\text { Headache } \\
\text { Pattern }\end{array}$ \\
\hline \multirow{3}{*}{ TLC } & $\begin{array}{l}\text { Abnormal }^{\mathrm{a}} \\
95(14.5)\end{array}$ & $-0.07(1.10)$ & $0.25(1.00)$ & $0.29(1.11)$ & $0.01(0.85)$ & $-0.14(0.87)$ & $-0.19(0.93)$ \\
\hline & $\begin{array}{l}\text { Normal } \\
562(85.5)\end{array}$ & $0.08(1.05)$ & $0.06(0.92)$ & $0.02(1.00)$ & $0.12(1.10)$ & $-0.02(0.93)$ & $-0.19(0.87)$ \\
\hline & Rho, $(p)^{\mathrm{b}}$ & $0.3(0.46)$ & $-0.4(0.29)$ & $-0.09(0.02)$ & $-0.06(0.16)$ & $0.08(0.06)$ & $0.05(0.25)$ \\
\hline \multirow{3}{*}{$\begin{array}{l}\text { FeV1 to FVC } \\
\text { ratio }\end{array}$} & $\begin{array}{l}\text { Abnormal }^{\mathrm{c}} \\
24(3.6)\end{array}$ & $0.12(1.05)$ & $-0.01(0.99)$ & $0.67(1.30)$ & $0.23(1.28)$ & $0.07(1.21)$ & $0.14(1.07)$ \\
\hline & $\begin{array}{l}\text { Normal } \\
643(96.4)\end{array}$ & $0.06(1.05)$ & $0.10(0.93)$ & $0.04(1.00)$ & $0.10(1.06)$ & $-0.04(0.93)$ & $-0.21(0.87)$ \\
\hline & Rho, $(p)^{\mathrm{b}}$ & $-0.16(<0.001)$ & $0.11(0.01)$ & $0.01(0.94)$ & $-0.15(<0.001)$ & $0.11(0.01)$ & $0.11(0.01)$ \\
\hline
\end{tabular}

Abbreviations: DLCO-Diffusing lung capacity of carbon monoxide; FeV1—Forced expiratory volume in $1 \mathrm{~s}$, FVC-forced vital capacity; TLC-Total lung capacity. ${ }^{a}$ Below $80 \%$ of expected; ${ }^{b}$ Spearman's correlation with the continuous variable; ${ }^{\mathrm{c}}$ A ratio of 0.7 and below.

\section{Discussion}

In this cohort of individuals with long COVID, most of whom were in a non-severe acute phase of the disease, we identified six patterns of symptoms, including cognitive; pain syndrome; pulmonary; cardiac; anosmia-dysgeusia; and headache. Each pattern was associated with distinct symptoms of the acute illness; and had different implications on the return to work and to everyday functionality.

Mechanisms presumably involved in tissue damage in COVID-19 include direct viral invasion of the tissues, disorders during acute infection (e.g., thromboembolism, microvascular damage), and post-infectious immune responses. All of these are considered possible mechanisms for persistent organ damage leading to long COVID symptoms and may involve a specific system (e.g., the nervous system) [10], which might explain clustering of symptoms (e.g., the cognitive-emotional-fatigue cluster/pattern).

The pulmonary pattern in our study was linked to prior lung disease and the acute disease severity (hospitalization and WHO classifications of severe and critical COVID-19) and is probably the result of damage to the lung parenchyma itself and the endothelium. This pattern is similar to sequelae described following severe acute respiratory syndrome (SARS), Middle East respiratory syndrome (MERS) and other causes of viral pneumonitis $[11,12]$. Disease severity was also linked to the pain-syndrome pattern, and may reflect, at least in part, a post-ICU syndrome, combining paresthesia and fatigue, which may represent muscle weakness [13].

Neuro-psychiatric sequelae were also reported following SARS and MERS; however, these were mostly post-traumatic stress disorder (PTSD) and neurological dysfunction following intensive care treatment $[14,15]$. In our cohort, the cognitive-emotional pattern was not related to the severity of the acute phase of the disease and, in fact, was seen more often in persons who were not hospitalized. We can raise different hypotheses as to the causes of the cognitive-emotional pattern: micro-thrombi, direct invasion of the brain by the virus or elevated cytokines [16]. Cognitive impairments could be induced or exaggerated by emotional disturbances, as has been consistently observed in various psychiatric disorders [17]. This pattern's association with failure to return to baseline employment status even at a median of four months following mild-moderate COVID-19, and its lack of association with time elapsed from the acute disease (hinting it does not decrease over time) suggests that this may become the true hallmark of long COVID burden on the individuum and the society.

Persistent fatigue, the main symptom described in all long COVID studies, was found to distribute equally in two main patterns (the cognitive and the pain syndrome patterns) and to trend highly in a third (the cardiac pattern). This finding is reminiscent of the two main examples of lasting fatigue: chronic fatigue syndrome and fibromyalgia $[15,18]$, sometimes attributed to viral illnesses such as Epstein-Barr virus, SARS and Chikungunya, among others [19]. Fatigue is the only symptom exhibiting this type of distribution among the patterns. This distribution may imply that there are multiple etiologies of fatigue 
(physical, mental, emotional) and that, therefore, fatigue should be researched according to the accompanying symptoms or more specific features.

The cardiac (palpitations-chest pain) pattern is suggestive of pericardial or myocardial involvement and, interestingly, was inversely associated with age, and prior diagnosis of atrial fibrillation, suggesting an immune or a dysregulatory process, rather than an ischemic one. Peri-myocarditis has been described as part of acute COVID and in post-COVID patients [20]. Myocardial injury was found in outpatients recovering from mild disease on magnetic resonance imaging (MRI) of the heart, regardless of ongoing symptoms [21]. It is yet to be determined whether this is a result of direct viral involvement or immunemediated damage.

Persistent anosmia and dysgeusia have been described and can be detrimental for patients' quality of life. Our findings support the hypothesis that the mechanism for this phenomenon is viral infection of the nasal epithelium [22], rather than generalized illness or viral brain invasion, which may explain its appearance as an isolated symptom independent of the neuro-psychiatric and other patterns.

Long COVID symptoms in children have also been reported, albeit to a lesser extent than in adults [23,24]. A similar factor analysis may be beneficial in the pediatric population as well.

This study has several limitations. First, the sample might not be representative of all patients with complaints following the acute disease; however, the symptoms correlation matrix was probably not biased by that. Second, the data were collected from four active COVID centers, three during a clinic visit and the fourth, a telephone interview; questioning patients in four different countries and different languages, using different interview methods might create an information bias. In addition, data regarding pre-COVID status was lacking, hampering comparisons of post-COVID data (such as pulmonary function tests and physical activity status). Lastly, patients were recruited at different intervals post-acute COVID with a median of four months. This reflects real life data but might affect the factor analysis, as the six patterns might behave differently over time. This is reflected in the finding that the pain-syndrome, pulmonary and cardiac patterns seem to wane over time.

Our study suggests implications for clinical management. In the framework of a multidisciplinary clinic, we recommend using a pattern-based approach, enabling management to focus on the most debilitating sets of symptoms in each patient. Patients suffering mainly from mental impairment would benefit from occupational rehabilitation and emotional support. Those with involvement of the lungs need dedicated follow-up and pulmonary rehabilitation. Patients with the cardiac pattern should be evaluated for risk of myocardial injury before returning to physical activity and those with anosmia-dysgeusia, fatigue and pain-syndrome patterns should be treated symptomatically and avoid unnecessary investigations. This is crucial given the colossal numbers of infected individuals and the limited capacity of health systems globally.

The study also has implications for research. The cognitive impairments are not explained by the severity of the acute disease; an effort should be made to identify the underlying mechanism, both through structural and functional imaging, and by investigating possible humoral explanations. Individuals with a predominant pulmonary pattern should be considered for clinical trials aimed at avoiding fibrotic disease and the cardiac pattern should be investigated in order to identify whether it is indeed rooted in cardiac injury and associated with long term impact on cardiovascular morbidity. Individuals suffering from the pain-syndrome pattern should be entered into long-term follow-up studies to identify optimal management strategies; patients with anosmia or dysgeusia should be evaluated for chemosensory research. This approach may enable an improved understanding of the pathophysiology and enable better allocation for future clinical trials. 
Supplementary Materials: The following supporting information can be downloaded at: https: / / www.mdpi.com/article/10.3390/jcm11040898/s1, Patient recruitment and data collection per country; Figure S1: Participant flow diagram; Table S1: Rotated Component Matrix-Table showing the loading of each symptom in each factor (pattern).

Author Contributions: Israel: L.L. and D.Y. (Dana Yelin) conceived the study idea, designed the study, planned the statistical analysis, verified the underlying data, and drafted the manuscript; E.W. conceived the study idea; I.M. designed the study and planned the statistical analysis, contributed to the underlying research protocol, data collection and writing the manuscript; I.S.-L., D.Y. (Dafna Yahav) contributed to the underlying research protocol and writing the manuscript; All authors contributed to manuscript revision, read and approved the submitted version. Switzerland: M.N. contributed to the underlying research protocol, collecting data and writing the manuscript; I.G. contributed to the underlying research protocol, collecting data and supervising the work in Switzerland; P.V. and L.K. contributed to the underlying research protocol, collecting data; All authors contributed to manuscript revision, read and approved the submitted version. Spain: C.G. contributed to the underlying research protocol, coordinating the study in Spain and writing the manuscript; J.B.-M. contributed to the underlying research protocol, collecting data and writing the manuscript; X.D.-M. and D.P.-C. contributed to the underlying research protocol and data collection; All authors read and approved the final version of the manuscript. Italy: M.F. contributed to the underlying research protocol, collecting data, writing the manuscript and supervising the work in Italy; G.T., L.C., and F.P. contributed to the underlying research protocol, collecting data and writing the manuscript. All participants of LongCOV research group contributed equally in data extraction. All authors have read and agreed to the published version of the manuscript.

Funding: This work was supported by the Israeli Insurance Association research fund, grant no. 050-351-010. The funding organization had no role in the planning, writing or analysis of the data in this study.

Institutional Review Board Statement: The study was conducted in accordance with the Declaration of Helsinki, and approved by the Institutional Review Boards (or Ethics Committees) of the 4 centers (approval numbers: Israel 0458-20-RMC; Italy CEAVNO n. 1768; Spain PR374/20; Switzerland CER 2020-01273).

Informed Consent Statement: All patients signed or gave oral informed consent prior to participation.

Data Availability Statement: Data will be available upon reasonable request from the corresponding author.

Acknowledgments: This study was performed as part of Dana Yelin's MPH thesis, Tel Aviv University. LongCOV study group members: Dorit Shitenberg, Tanya Babich, Talya Kalfon; Michal Mor; Yair Shafir, Hadar Agmon, Ori Tishler, Donna Abecasis, Nitzan Karny-Epstein, Alaa Atamna, Vered Daitch, Laura Tavanti, Davide Chimera, Antonio Fideli, Margherita Biagini, Alessandra Pagano, Roberta Pancani, Massimiliano Desideri, Nicoletta Carpenè, Luciano Gabbrielli, Maurizio Lucchesi, Alessandro Celi, Micol Micheli, Lorenzo Ghiadoni, Greta Barbieri, Chiara Romei, Annalisa Deliperi, Fabio Monzani, Ferruccio Aquilini, Michele Cristofano, Valentina Galfo, Francesco Menichetti, Chiara Barbieri, Alba Bergas, Vanessa Vicens-Zygmunt, Cristina Orive-Lago, Meritxell Regi-Bosque, Ana Peñalver-Garcia, Antonia Requesens-Catalan, Nuria Perez-Gutiérrez, Giovanna Miranda, Barbara Navarro, Eva Alonso-Bes, Olga Bella, Enilda Cordero, Elena Carrera-Izquierdo, Sonia Martínez-Carmona.

Conflicts of Interest: The authors declare no conflict of interest.

\section{References}

1. Sudre, C.H.; Murray, B.; Varsavsky, T.; Graham, M.S.; Penfold, R.S.; Bowyer, R.C.; Pujol, J.C.; Klaser, K.; Antonelli, M.; Canas, L.S.; et al. Attributes and predictors of long COVID. Nat. Med. 2021, 27, 626-631. [CrossRef] [PubMed]

2. Townsend, L.; Dyer, A.H.; Jones, K.; Dunne, J.; Mooney, A.; Gaffney, F.; O'Connor, L.; Leavy, D.; O’Brien, K.; Dowds, J.; et al. Persistent fatigue following SARS-CoV-2 infection is common and independent of severity of initial infection. PLoS ONE 2020, 15, e0240784. [CrossRef] [PubMed]

3. Bliddal, S.; Banasik, K.; Pedersen, O.B.; Nissen, J.; Cantwell, L.; Schwinn, M.; Tulstrup, M.; Westergaard, D.; Ullum, H.; Brunak, S.; et al. Acute and persistent symptoms in non-hospitalized PCR-confirmed COVID-19 patients. Sci. Rep. 2021, 11, 13153. [CrossRef] [PubMed] 
4. Peghin, M.; Palese, A.; Venturini, M.; De Martino, M.; Gerussi, V.; Graziano, E.; Bontempo, G.; Marrella, F.; Tommasini, A.; Fabris, M.; et al. Post-COVID-19 symptoms 6 months after acute infection among hospitalized and non-hospitalized patients. Clin. Microbiol. Infect. 2021, 27, 1507-1513. [CrossRef] [PubMed]

5. Carvalho-Schneider, C. Follow-up of adults with non-critical COVID-19 two months after symptoms' onset. Clin. Microbiol. Infect. 2020, 27, 258-263. [CrossRef] [PubMed]

6. Del Rio, C.; Collins, L.F.; Malani, P. Long-term Health Consequences of COVID-19. JAMA 2020, 324, 1723-1724. [CrossRef]

7. Yelin, D.; Wirtheim, E.; Vetter, P.; Kalil, A.C.; Bruchfeld, J.; Runold, M.; Guaraldi, G.; Mussini, C.; Gudiol, C.; Pujol, M.; et al. Long-term consequences of COVID-19: Research needs. Lancet Infect. Dis. 2020, 20, 1115-1117. [CrossRef]

8. Nehme, M.; Braillard, O.; Chappuis, F.; Courvoisier, D.S.; Guessous, I. Prevalence of Symptoms More Than Seven Months After Diagnosis of Symptomatic COVID-19 in an Outpatient Setting. Ann. Intern. Med. 2021, 174, 1252-1260. [CrossRef]

9. WHO. WHO Interim Clinical Guidance May 27 2020. Available online: https://www.who.int/publications/i/item/clinicalmanagement-of-covid-19 (accessed on 28 April 2021).

10. Scoppettuolo, P.; Borrelli, S.; Naeije, G. Neurological involvement in SARS-CoV-2 infection: A clinical systematic review. Brain Behav. Immun. Health 2020, 5, 100094. [CrossRef]

11. Zhang, P.; Li, J.; Liu, H.; Han, N.; Ju, J.; Kou, Y.; Chen, L.; Jiang, M.; Pan, F.; Zheng, Y.; et al. Long-term bone and lung consequences associated with hospital-acquired severe acute respiratory syndrome: A 15-year follow-up from a prospective cohort study. Bone Res. 2020, 8, 8. [CrossRef]

12. Kim, E.A.; Lee, K.S.; Primack, S.L.; Yoon, H.K.; Byun, H.S.; Kim, T.S.; Suh, G.Y.; Kwon, O.J.; Han, J. Viral Pneumonias in Adults: Radiologic and Pathologic Findings. RadioGraphics 2002, 22, S137-S149. [CrossRef] [PubMed]

13. Neufeld, K.J.; Leoutsakos, J.-M.S.; Yan, H.; Lin, S.; Zabinski, J.S.; Dinglas, V.D.; Hosey, M.M.; Parker, A.M.; Hopkins, R.O.; Needham, D.M. Fatigue Symptoms During the First Year Following ARDS. Chest 2020, 158, 999-1007. [CrossRef]

14. Hui, D.S.C.; Wong, K.T.; Antonio, G.E.; Tong, M.; Chan, D.P.; Sung, J.J.Y. Long-term sequelae of SARS: Physical, neuropsychiatric, and quality-of-life assessment. Hong Kong Med. J. 2009, 15, 21-23. [PubMed]

15. Lam, M.H.-B.; Wing, Y.-K.; Yu, M.W.-M.; Leung, C.-M.; Ma, R.C.W.; Kong, A.P.S.; So, W.; Fong, S.Y.-Y.; Lam, S.-P. Mental Morbidities and Chronic Fatigue in Severe Acute Respiratory Syndrome Survivors: Long-term Follow-up. Arch. Intern. Med. 2009, 169, 2142-2147. [CrossRef] [PubMed]

16. Reichenberg, A.; Yirmiya, R.; Schuld, A.; Kraus, T.; Haack, M.; Morag, A.; Pollmächer, T. Cytokine-Associated Emotional and Cognitive Disturbances in Humans. Arch. Gen. Psychiatry 2001, 58, 445-452. [CrossRef]

17. Abramovitch, A.; McCormack, B.; Brunner, D.; Johnson, M.; Wofford, N. The impact of symptom severity on cognitive function in obsessive-compulsive disorder: A meta-analysis. Clin. Psychol. Rev. 2018, 67, 36-44. [CrossRef] [PubMed]

18. Hickie, I.; Davenport, T.; Wakefield, D.; Vollmer-Conna, U.; Cameron, B.; Vernon, S.D.; Reeves, W.C.; Lloyd, A. Post infective and chronic fatigue syndromes precipitated by viral and non-viral pathogens: Prospective cohort study. BMJ 2006, $333,575$. [CrossRef] [PubMed]

19. Buchwald, D.; Ashley, R.L.; Pearlman, T.; Kith, P.; Komaroff, A.L. Viral serologies in patients with chronic fatigue and chronic fatigue syndrome. J. Med. Virol. 1996, 50, 25-30. [CrossRef]

20. Imazio, M.; Klingel, K.; Kindermann, I.; Brucato, A.; De Rosa, F.G.; Adler, Y.; De Ferrari, G.M. COVID-19 pandemic and troponin: Indirect myocardial injury, myocardial inflammation or myocarditis? Heart 2020, 106, 1127-1131. [CrossRef]

21. Puntmann, V.O.; Carerj, M.L.; Wieters, I.; Fahim, M.; Arendt, C.; Hoffmann, J.; Shchendrygina, A.; Escher, F.; Vasa-Nicotera, M.; Zeiher, A.M.; et al. Outcomes of Cardiovascular Magnetic Resonance Imaging in Patients Recently Recovered from Coronavirus Disease 2019 (COVID-19). JAMA Cardiol. 2020, 5, 1265-1273. [CrossRef]

22. Zheng, J.; Wong, L.-Y.R.; Li, K.; Verma, A.K.; Ortiz, M.E.; Wohlford-Lenane, C.; Leidinger, M.R.; Knudson, C.M.; Meyerholz, D.K.; McCray, P.B., Jr.; et al. COVID-19 treatments and pathogenesis including anosmia in K18-hACE2 mice. Nature 2021, 589, 603-607. [CrossRef] [PubMed]

23. Ashkenazi-Hoffnung, L.; Shmueli, E.; Ehrlich, S.; Ziv, A.; Bar-On, O.; Birk, E.; Lowenthal, A.; Prais, D. Long COVID in Children: Observations from a Designated Pediatric Clinic. Pediatr. Infect. Dis. J. 2021, 40, e509-e511. [CrossRef] [PubMed]

24. Buonsenso, D.; Munblit, D.; De Rose, C.; Sinatti, D.; Ricchiuto, A.; Carfi, A.; Valentini, P. Preliminary evidence on long COVID in children. Acta Paediatr. 2021, 110, 2208-2211. [CrossRef] [PubMed] 